\title{
AUTHOR INDEX VOLUME 18
}

Ågren H, see Fu $Y$

18 (2009) 195

Ahmad H, see Shahi $S$

18 (2009) 521

Al-Naimee KA, see Kbashi HJ

18 (2009) 541

Ali J, see Hanim NF

Andrews DL, see Bradshaw DS

18 (2009) 481

18 (2009) 285

Assanto G, Minzoni AA \& Smyth NF, Light Self-Localization in Nematic Liquid Crystals: Modelling Solitons in Nonlocal Reorientational Media

18 (2009) 657

Barna V, see De Luca A

18 (2009) 349

Bartolino R, see De Luca A

18 (2009) 349

Basque V, see Riordon $J$

18 (2009) 693

Batani D, see Kbashi HJ

18 (2009) 541

Benocci R, see Kbashi HJ

18 (2009) 541

Biswas A, see Kohl $R$

Bradshaw DS \& Andrews DL, Quantum Channels in Nonlinear Optical Processes

18 (2009) 227

Brasselet E \& Juodkazis S, Optical Angular Manipulation of Liquid Crystal Droplets in Laser Tweezers

Cai S, see $X u H$

Caputo R, see De Luca A

Carpeggiani P, see Kbashi HJ

Chan CP, Lam H, Leung KK \& Surya C, Growth of Copper Zinc Tin

18 (2009) 285

18 (2009) 167

18 (2009) 141

18 (2009) 349

18 (2009) 541

Sulfide Nano-Rods by Electrodeposition Using Anodized Aluminum as the Growth Mask

18 (2009) 599

Chattopadhyay T, All-Optical Terahertz Optical Asymmetric

Demultiplexer (TOAD) Based Binary Comparator: A Proposal

Chen $\mathrm{G}$, see Hao $Z$

Chen G, see Liang $H$

Chen X, Liu K \& Shi J, Polarization Coupling of Light Waves in Periodically Poled Lithium Niobate and Its Applications

Cheng C, see Tang $Y$

Cheng X, see Zhu $Y$

Cheng X, Zhuang B, Dai X, Su W \& Wen S, Dark Soliton Solutions to the Nonlinear Schrödinger Equation for Ultrashort Pulse

Propagation in Metamaterials

18 (2009) 471

18 (2009) 327

18 (2009) 605

18 (2009) 49

18 (2009) 309

18 (2009) 319

Clays K, see Pérez-Moreno J

18 (2009) 271

18 (2009) 401

Dai J, see Guo Z

18 (2009) 617

Dai J, see Guo Z

18 (2009) 625

Dai X, see Cheng $X$

18 (2009) 271 
De Luca A, Barna V, Ferjani S, Caputo R, Versace C, Scaramuzza N, Bartolino R, Umeton C \& Strangi G, Laser Action in Dye Doped Liquid Crystals: From Periodic Structures to Random Media

Ding W, see Guo Z

Dong P, Tian Y, Xiao X \& Yang C, Wavelength Conversion of Multichannel Spectrum-Sliced ASE Signals via Four-Wave Mixing in Highly Nonlinear Dispersion-Shifted Fiber

Dorsinville $\mathrm{R}$, see Ortega A

Escobedo-A J, see Grimalsky $V$

Ferjani S, see De Luca A

Finot C \& Wu L, Influence of the Initial Phase Profile on the Asymptotic Self-Similar Parabolic Dynamics

Fu M, Zhao A, Zhou J, He D \& Wang Y, Morphologically Controlled Electrodeposition of $\mathrm{ZnO}$ and $\mathrm{Cu}_{2} \mathrm{O}$ by the Colloidal Crystal Template Method

Fu Y, Hellström S \& Ågren H, Nonlinear Optical Properties of Quantum Dots: Excitons in Nanostructures

Fuh AY-G, see Lin $H-C$

Gao S, see Gao Y

Gao Y \& Gao S, Premodulation-Free Microwave Frequency Up/Down-Conversion Using Optical-Fiber-Stimulated Brillouin Scattering

Grimalsky V, Koshevaya S \& Escobedo-A J, Interaction of Infrared Electromagnetic Waves in Resonant Layered Structures with n-GaAs Semiconductor Film

Guo S, see Zhu $Y$

Guo Z, Ding W, Qu S, Dai J \& Liu S, Self-Assembled Volume Grating in Silica Glass Induced by Tightly Focused Femtosecond Laser Pulse

Guo Z, Wang H, Liu Z, Qu S, Dai J \& Liu S, Realization of Holographic Storage on Metal Film by Femtosecond Laser Pulses Micromachining

Guo-Li S, Luminescence Characteristics of Rare-Earth Erbium Ion-Doped Nanocrystalline Zinc Oxide

Haché A, see Riordon J

Han L, see Liu $Y$

Han L, see Liu $Y$

Hanim NF, Saktioto Ali J \& Rahman RA, Modeling of Induced Voltage of Silicon Dioxide Fiber Coupling

18 (2009) 349

18 (2009) 625

18 (2009) 341

18 (2009) 161

18 (2009) 73

18 (2009) 349

18 (2009) 709

18 (2009) 611

18 (2009) 195

18 (2009) 367

18 (2009) 701

18 (2009) 701

18 (2009) 73

18 (2009) 319

18 (2009) 625

18 (2009) 617

18 (2009) 649

18 (2009) 693

18 (2009) 553

18 (2009) 561

18 (2009) 481

Hao Z, Lu W, Zhang C, Tian J, Chen G, Xu T \& Qi S, Laser Induced Absorption Spectra Properties of Ethyl Red Doped Film and Its Potential Applications for Optical Switch Based on Two Beams Mutual Modulation

18 (2009) 327

18 (2009) 521

18 (2009) 611

He D, see Fu $M$

He YJ, Luo L, Zhu WL \& Wang HZ, Formation and Collision of $(1+1)$-Dimensional Dipole Solitons in the Strongly Non-Local Non-Linear Media

18 (2009) 121

Hellström S, see Fu Y

18 (2009) 195

Hoai TX, see Tung NT

18 (2009) 489

Jawad H, see Kbashi HJ

18 (2009) 541 
Jia B, see Liu $Y$

18 (2009) 553

Jia B, see Liu $Y$

Juodkazis S, see Brasselet $E$

Kaur G, Singh ML \& Patterh MS, Investigations of Fiber

18 (2009) 561

18 (2009) 167

Nonlinearities in Long-Haul Optical WDM Transmission Systems

Kaur G, Singh ML \& Patterh MS, Theoretical Investigations to

18 (2009) 301

Minimize Bit Error Rate by Optimizing System Parameters in

Optical DWDM Transmission Systems at Different Data Rates

Kbashi HJ, Jawad H, Al-Naimee KA, Benocci R, Carpeggiani P,

Perego C \& Batani D, Spectral Width Variation of Ultrashort Laser

Pulses in Monomode Optical Fibers

Kellou A, see Mallek D

Kheradmand R, see Sahrai $M$

Kim DY, see Song $H$

Kimball BR, see Nersisyan SR

Kirubavathi K, Selvaraju K \& Kumararaman S, Studies on Growth and Characterization of Bis Thiourea Lead Chloride: A Novel Nonlinear Optical Crystal

18 (2009) 501

18 (2009) 541

18 (2009) 111

18 (2009) 529

18 (2009) 99

18 (2009) 1

18 (2009) 153

Kohl R, Milovic D, Zerrad E \& Biswas A, Soliton Perturbation Theory for Dispersion-Managed Optical Fibers

Koshevaya S, see Grimalsky $V$

Kumararaman S, see Kirubavathi $K$

Lam H, see Chan $C P$

Lam VD, see Tung NT

Leblond $\mathrm{H}$, see Mallek $D$

Lee YP, see Tung NT

Leung KK, see Chan CP

Li L, see Liang $H$

Liang H, Wu L, Chen G, Liu Y, Li L \& Zhang Z, Ultraviolet and Blue Upconversion Emissions of $\mathrm{NaYF}_{4}: \mathrm{La}^{3+}\left(\mathrm{Er}^{3+}, \mathrm{Tb}^{3+}\right)$

Nanocrystals Under $532 \mathrm{~nm}$ Laser Excitation

Lin H-C \& Fuh AY-G, Z-Scan Measurements of Optical Nonlinearities of Dye-Doped Liquid Crystals

Lipsanen H, see Mustonen A

Liu C, see Shen $X$

Liu K, see Chen $X$

Liu S, see Guo Z

Liu S, see Guo Z

Liu Y, Lu W, Yu Z, Jia B, Xu Z, Lu P \& Han L, The Strain Field Distribution of Quantum Dot Array with Conical Shape

Liu Y, see Liang $H$

Liu Y, Xu Z, Yu Z, Jia B, Lu W, Lu P \& Han L, Strain Distribution and Electronic Structure of Self-Organized InAs/GaAs Quantum Dots

Liu Z, see Guo Z

Lu P, see Liu $Y$

Lu P, see Liu $Y$

$\mathrm{Lu}$ Q, see Zhu $Y$

$\mathrm{Lu} \mathrm{W}$, see Hao $Z$

Lu W, see Liu $Y$

18 (2009) 227

18 (2009) 73

18 (2009) 153

18 (2009) 599

18 (2009) 489

18 (2009) 111

18 (2009) 489

18 (2009) 599

18 (2009) 605

18 (2009) 605

18 (2009) 367

18 (2009) 633

18 (2009) 465

18 (2009) 49

18 (2009) 617

18 (2009) 625

18 (2009) 561

18 (2009) 605

18 (2009) 553

18 (2009) 617

18 (2009) 553

18 (2009) 561

18 (2009) 319

18 (2009) 327

18 (2009) 553 
Lu W, see Liu $Y$

$\mathrm{Lu} \mathrm{YH}$, see Zhang $H X$

Luo L, see He YJ

Ma L, see Zhang $Y$

Mahmoudi M, see Sahrai $M$

Makwani D \& Vijaya R, Solvent Effects on the Dispersion of Linear Polarizability and First Hyperpolarizability of Para-Nitroaniline Using Polarizable Continuum Model

Mallek D, Kellou A, Leblond H \& Sanchez F, Brillouin Instabilities in Continuously Pumped High Power Fiber Lasers

Milovic D, see Kohl $R$

Minzoni AA, see Assanto $G$

Moghaddam MRA, see Shahi $S$

Mustonen A \& Lipsanen H, Fabrication and Optical Properties of Metal-Coated Non-Close-Packed Colloidal Crystals

Nersisyan SR, Tabiryan NV, Steeves DM \& Kimball BR, Optical Axis Gratings in Liquid Crystals and Their Use for Polarization

Insensitive Optical Switching

Ogawa T, see Ortega A

Ortega A, Perez-Martinez AL, Ogawa T, Smith F, Walser A \& Dorsinville R, Third-Order Nonlinear Optical Property of Poly(Hexa-2,4-Diynylene-1,6-Dioxydicinnamate) Containing a Polar Azo Dye, Determined by Z-Scan Technique

Paek U-C, see Song $H$

Patterh MS, see Kaur $G$

Patterh MS, see Kaur $G$

Perego C, see Kbashi HJ

Perez-Martinez AL, see Ortega A

Pérez-Moreno J \& Clays K, Fundamental Limits: Developing New Tools for a Better Understanding of Second-Order Molecular Nonlinear Optics

Qi S, see Hao Z

$\mathrm{Qu} \mathrm{S}$, see Guo Z

$\mathrm{Qu} \mathrm{S}$, see Guo Z

Rahman RA, see Hanim NF

Riordon J, Basque V \& Haché A, Broadband Pump-Probing Inside an Optical Parametric Amplifier

Sahrai M, Kheradmand R, Salahshour N \& Mahmoudi M, The Effect of Decay-Induced Interference on the Phase Control of the Optical Bistability $(O B)$

Saktioto see Hanim NF

Salahshour N, see Sahrai $M$

Sanchez F, see Mallek $D$

Scaramuzza N, see De Luca A

Selvaraju K, see Kirubavathi $K$

Seo J, see Yang $Q$

Sergio G, see Tang $Y$

Shahi S, Moghaddam MRA, Harun SW \& Ahmad H, The Comparison Nonlinearity Behaviors of Photonic Crystal Fiber by Two Reduced Lengths of Bi-EDF in Ring Cavity

18 (2009) 561

18 (2009) 441

18 (2009) 121

18 (2009) 583

18 (2009) 529

18 (2009) 85

18 (2009) 111

18 (2009) 227

18 (2009) 657

18 (2009) 521

18 (2009) 633

18 (2009) 1

18 (2009) 161

18 (2009) 161

18 (2009) 99

18 (2009) 301

18 (2009) 501

18 (2009) 541

18 (2009) 161

18 (2009) 401

18 (2009) 327

18 (2009) 617

18 (2009) 625

18 (2009) 481

18 (2009) 693

18 (2009) 529

18 (2009) 481

18 (2009) 529

18 (2009) 111

18 (2009) 349

18 (2009) 153

18 (2009) 573

18 (2009) 309

18 (2009) 521 
Shen H, see Shen $X$

18 (2009) 465

Shen X, Liu C \& Shen H, Nonlinear Optical Absorption in Ordered

18 (2009) 465

Au Nanoparticle Arrays

Shen Y, see $X u H$

18 (2009) 141

Shi J, see Chen $X$

Siahmakoun A, see Tang $Y$

Singh ML, see Kaur $G$

Singh ML, see Kaur $G$

Smith F, see Ortega $A$

Smyth NF, see Assanto $G$

Song H, Paek U-C \& Kim DY, Walk-Off Length Limited Spectral Broadening in Supercontinuum Generation

Steeves DM, see Nersisyan SR

Strangi G, see De Luca A

$\mathrm{Su}$ W, see Cheng $X$

Surya C, see Chan $C P$

Suryanto A, On the Numerical Modeling of Optical-Switching in Non-Linear Phase-Shifted Grating

Tabibi B, see Yang $Q$

Tabiryan NV, see Nersisyan SR

Tan G, see Yang $Q$

Tang Y, see Zheng $L$

Tang Y, Siahmakoun A, Sergio G, Teferra S, Vlahovic B \& Cheng C,

18 (2009) 49

18 (2009) 309

18 (2009) 301

18 (2009) 501

18 (2009) 161

18 (2009) 657

18 (2009) 99

18 (2009) 1

18 (2009) 349

18 (2009) 271

18 (2009) 599

18 (2009) 129

18 (2009) 573

18 (2009) 1

18 (2009) 573

18 (2009) 457

A Wavelength Conversion Based on Cross-Gain Modulation of a Semiconductor Optical Amplifier Fiber Ring Loop

Teferra S, see Tang $Y$

Temple D, see Yang $Q$

Tian H, see Zhang $J$

Tian J, see Hao Z

Tian Y, see Dong P

Tung NT, Lee YP, Hoai TX \& Lam VD, Impact of Geometrical Parameters on Transmission Properties of Cut-Wire Pair Structures

Umeton C, see De Luca A

Versace C, see De Luca A

Vijaya R, see Makwani D

Vlahovic B, see Tang $Y$

Walser A, see Ortega A

Wang H, see Guo Z

Wang HZ, see He YJ

Wang J, see Zhang $J$

Wang N, see Zhang $J$

18 (2009) 309

18 (2009) 309

18 (2009) 573

18 (2009) 591

18 (2009) 327

18 (2009) 341

Wang Y, see Fu $M$

Wen $\mathrm{S}$, see Cheng $X$

Wu B, see $X u H$

$\mathrm{Wu} \mathrm{D}$, see Zhang $J$

Wu L, see Finot $C$

Wu L, see Liang $H$

Xiao X, see Dong P

18 (2009) 489

18 (2009) 349

18 (2009) 349

18 (2009) 85

18 (2009) 309

18 (2009) 161

18 (2009) 617

18 (2009) 121

18 (2009) 591

18 (2009) 591

18 (2009) 611

18 (2009) 271

18 (2009) 141

18 (2009) 591

18 (2009) 709

18 (2009) 605

18 (2009) 341 
$\mathrm{Xu} \mathrm{H}, \mathrm{Wu} \mathrm{B}, \mathrm{Cai} \mathrm{S} \&$ Shen Y, Investigation on the Pump Acceptance Bandwidth for Collinear Quasi-Phase-Matching Optical Parametric Amplification

18 (2009) 141

$\mathrm{Xu} \mathrm{T}$, see Hao $Z$

18 (2009) 327

$\mathrm{Xu} \mathrm{Z}$, see Liu $Y$

18 (2009) 553

18 (2009) 561

$\mathrm{Xu} Z$, see Liu $Y$

Yang C, see Dong $P$

Yang C, see Zhang $Y$

18 (2009) 341

18 (2009) 583

Yang Q, Seo J, Tabibi B, Yu W, Tan G \& Temple D, Time Domain Description of the Group Velocity Manipulation Using Semiconductor Quantum Dots

Yu B, see Zhang $J$

Yu B \& Zhang Y, Flatband Slow Light Photonic Crystal Waveguide in Both TE and TM Polarizations

$\mathrm{Yu} \mathrm{W}$, see Yang $Q$

$\mathrm{Yu} Z$, see Liu $Y$

Yu Z, see Liu $Y$

Yuan P, see Zhang $J$

Yuan P, see Zhang $Y$

Zerrad E, see Kohl $R$

Zhang C, see Hao Z

Zhang HX, Zhao L \& Lu YH, Study on a Sort of Controllable

Nonlinear Left-Handed Materials

Zhang J, Zhang Y, Zhang X, Yu B, Wu D, Tian H, Yuan P, Wang N \& Wang J, Slow Light in a Two Microsphere Optical Fiber System Zhang X, see Zhang $J$

Zhang Y, Ma L, Yang C \& Yuan P, Nonlinear-Optical and Optical Limiting Properties of Phenoxy-Phthalocyanines Studied Using the Z-Scan Technique

Zhang $\mathrm{Y}$, see $Y u B$

Zhang Y, see Zhang $J$

Zhang Z, see Liang $H$

Zhao A, see Fu $M$

Zhao L, see Zhang $H X$

Zheng L \& Tang Y, Energy Exchange of Interacting Dissipative Optical Bullets in Dissipative System

Zhou J, see Fu $M$

Zhu WL, see He YJ

Zhu Y, Lu Q, Guo S \& Cheng X, Threshold Reduction of Stimulated Brillouin Scattering by Enhancing Initial Brillouin Ultrasounds

Zhuang B, see Cheng $X$

18 (2009) 573

18 (2009) 591

18 (2009) 641

18 (2009) 573

18 (2009) 553

18 (2009) 561

18 (2009) 591

18 (2009) 583

18 (2009) 227

18 (2009) 327

18 (2009) 441

18 (2009) 591

18 (2009) 591

18 (2009) 583

18 (2009) 641

18 (2009) 591

18 (2009) 605

18 (2009) 611

18 (2009) 441

18 (2009) 457

18 (2009) 611

18 (2009) 121

18 (2009) 319

18 (2009) 271 